\title{
Classification of Kinematic Swimming Data with Emphasis on Resource Consumption
}

\author{
Ulf Jensen, Franziska Prade, and Bjoern M. Eskofier \\ Digital Sports Group, Pattern Recognition Lab, Department of Computer Science, University Erlangen-Nuremberg, Germany \\ Corresponding author: ulf.jensen@cs.fau.de
}

\begin{abstract}
The collection of kinematic data with a head-worn sensor is a promising approach for swimming data analysis in the context of athlete support systems. We present a new approach of analyzing these data and describe a system that segments the lanes of a swimming session and classifies the swimming style of each lane. Special emphasis was put on the algorithm efficiency and the analysis of the resource demands to be able to port the implementation to an embedded microcontroller. For developing the system, data of twelve subjects was collected. The data incorporated two different turn styles that mark the end of a lane as well as the four main swimming styles backstroke, breaststroke, butterfly and freestyle. All turns were successfully identified from the turn detection. Our fully automatic swimming style classification reached a classification rate of $95.0 \%$. The results from the resource consumption analysis can be used to support the decision for the embedded target hardware of a headworn swimming training system.
\end{abstract}

\section{INTRODUCTION}

Inertial sensor data is an important modality in state-of-theart swimming analysis. Compared to camera-based systems, sensor solutions enable long-term monitoring, provide usercentric analysis and do not suffer from water turbulences and image blurring. Advanced sensor systems for precise technique analysis were developed and proved to be helpful in high performance practice [1]-[3]. However, these systems suffer from enhanced water resistance and a complicated setup. Beside these systems, minimal sensor solutions that consist of wristwatch devices equipped with inertial sensors were proposed. These devices limit the comfort of the athlete and feedback on the wristwatch can only accessed in breaks.

This articles investigates an unobtrusive sensor position at the occiput for swimming data analysis capabilities. Kinematic sensor data of a single sensor were collected to develop an analysis system capable of detecting starting and turning events as well as classifying the four common swimming styles. Special emphasis was put on the computational complexity to enable an implementation on an embedded microcontroller or Body Area Sensor (BSN) node. Our approach provides an alternative to the wristwatch positioning and is the first step to an unobtrusive athlete support system for swimming.

Research on sensor-based swimming monitoring was first presented by Oghi et al. [4] in 2002, who presented a wristwatch accelerometer device. Since then, numerous studies used video- and accelerometer-based analysis to investigate swimming performance. A good overview and a comparison of both approaches can be found in [5]. Recently, a comprehensive swimming technique evaluation system including feedback mechanism was developed [2]. However, the data processing was performed offline and no instant feedback was given during exercise. The single unobtrusive sensor position on the head was first investigated in 2010 [6]. The authors proved to be able to detect three swimming styles, certain events like wall push-offs and turns as well as important parameters like stroke count and stroke duration. Though, results are only shown exemplary for one subject, not proven in a scientific study and only three of the four main swimming styles were investigated. Additionally, algorithms were not designed for an embedded real-time implementation. From a product perspective one of the most popular devices is the Pool Mate (Swimovate Ltd., Wraysbury, UK) wristwatch. It detects turns and strokes of the four main stroke types and can therefore be used as a lap counter, training tracker and efficiency monitor.

Our works overcomes some of the limitations in [6] and provides an alternative data analysis approach with pattern recognition methods. We conducted a research study to provide a head-worn swimming analysis system capable of detecting the state of the swimmer, turning events and the four main swimming styles. Special emphasis was put on the algorithm design to address limited hardware capabilities of an embedded microcontroller. The proposed system provides an alternative to the common inconvenient sensor position on the wrist and has the potential to be directly integrated in the swimming goggles to display training feedback in an integrated display.

\section{METHODS}

\section{A. Data collection}

1) Hardware: We used the SHIMMER ${ }^{\mathrm{TM}}$ sensor platform [7] to collect inertial sensor data. The main board includes a three-axis accelerometer and was extended with a three-axis gyroscope resulting in raw data of six dimensions. We set the sampling rate to $200 \mathrm{~Hz}$ and chose an accelerometer range of $\pm 1.5 \mathrm{~g}$. The gyroscope collected rotational velocity in the range of $\pm 500 \mathrm{deg} / \mathrm{sec}$. The sensor was waterproofed and data were logged on SD card.

The sensor node was placed on the occiput of the subject underneath the swimming cap. The position and the corresponding axes are schematically shown in Fig. 1.

2) Study design: We set up a research study to collect data from different states, events and swimming styles occurring during a swimming session. The four main swimming styles butterfly, backstroke, breaststroke and freestyle were considered. We defined an active and a resting state describing whether the athlete is swimming or not. As events, we collected flip and tumble turns at the end of a lane. The protocol of a session is shown in Tab. I and was based on the normal medley race order.

For data collection, 12 german $2^{\text {nd }}$ league swimmers ( 5 female, 


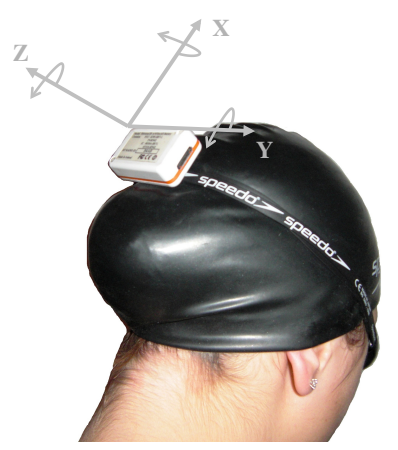

Fig. 1. Schematic sensor placement at subjects' occiput with accelerometer and gyroscope axes $\mathrm{x}, \mathrm{y}$ and $\mathrm{z}$.

7 male) aged 16-20 were recruited. After warm-up, subjects were asked to swim a $200 \mathrm{~m}$ medley distance with $80 \%$ of their personal best. We repeated the recording session at the end of a two hour training to get a variation in the swimming technique due to fatigue. Overall, eleven dataset with two sessions were recorded. Due to sensor malfunction, a single session at the beginning of the training session was collected for one subject.

TABLE I. PROTOCOL OF A 200 M MEDLEY SESSION WITH CORRESPONDING EVENTS AND SWIMMING STYLES

\begin{tabular}{rlll} 
Nr. & Description & Abbr. & Type \\
\hline 1 & Waiting at the poolside & REST & State \\
2 & Butterfly lane & SWIM & Interval \\
3 & Turn & TURN & Event \\
4 & Backstroke lane & SWIM & Interval \\
5 & Turn & TURN & Event \\
6 & Breaststroke lane & SWIM & Interval \\
7 & Turn & TURN & Event \\
8 & Freestyle lane & SWIM & Interval \\
9 & Resting at the poolside & REST & State \\
\hline
\end{tabular}

\section{B. Classification system}

Pattern recognition is an established methodology for highdimensional data analysis in biomedical [8] and sports applications [9]. We used pattern recognition methods for turn detection and swimming style classification. The simplified processing pipeline [10] of a classification system consists of the working phase and the training phase (Fig. 2). To classify unknown data, the working phase of the system is executed and will be described in the following section.

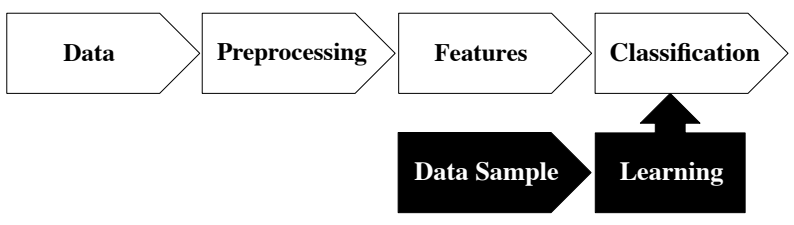

Fig. 2. Classification processing pipeline consisting of working phase (white) and training phase (black).

1) Data: The inertial sensor input for the classification system consisted of the 12-Bit analog-digital converter output.

2) Preprocessing: The SHIMMER calibration procedure described in the user manual [11] was used to convert the
TABLE II. COMPLETE FEATURE SET USED IN THE PROPOSED DATA ANALYSIS SYSTEM

\begin{tabular}{rlrl} 
Nr. & Feature & Nr. & Feature \\
\hline 1 & Mean & 5 & Kurtosis \\
2 & Standard Deviation & 6 & Skewness \\
3 & Variance & 7 & Minimum \\
4 & Energy & 8 & Maximum \\
\hline
\end{tabular}

raw analog-digital-converter output to acceleration and angular velocity units.

3) Features: We adopted a feature set consisting of statistical measures and signal characteristics which was previously evaluated for physiological and kinematic data [12]. The complete list of features is shown in Tab. II.

These eight features were computed for each axis of the input data. As six axes were recorded, this results in patterns of dimension 48 for each classification problem.

4) Classification: We implemented a classifier that takes a class decision based on linear regression models [13]. For each class $C$, a linear data model

$$
f_{C}(\mathbf{x})=\mathbf{w}^{\mathbf{T}} \mathbf{x}+w_{0}
$$

with weight vector $\mathbf{w}^{\mathbf{T}}$, constant $w_{0}$ and unknown feature vector $\mathrm{x}$ was estimated with linear regression in the training phase. Subsequently, in the working phase, the model of each class was evaluated. The results were transformed to probabilities as values bigger than one were set to one and negative values were set to zero. Furthermore, all values were scaled to sum up to one. The class whose function $f_{C}(\mathbf{x})$ delivered the highest probability was chosen as classification decision for the unknown pattern.

5) Evaluation: The Embedded Classification Software Toolbox (ECST) [14] was used to perform training and performance estimation of the classification system. In addition, the software is capable of computing the memory consumption and demand of mathematical operations for a classification system. It can therefore give an interesting benchmark for estimating the resource demands on the target hardware.

\section{Data processing}

1) State detection: The purpose of the state detection was to decide wether the athlete was swimming or resting. We considered two criteria for this decision, the signal energy and the head position (Fig. 3).

The signal energy defined a general level of head movements. After differentiating and taking the absolute value, we computed the signal energy $E(\mathbf{x})$ of each sample $\mathbf{t}$ using

$$
E(\mathbf{t})=\mathbf{w}^{\mathbf{T}} \mathbf{t}
$$

We incorporated a set of weights $\mathbf{w}$ in the computation for two reasons. First, to account for the influence of the two different sensor types, as the acceleration was in the numerical range of \pm 1.5 and the gyroscope in the numerical range of \pm 500 . Second, to attenuate axes that best reflected the state differences. All weights were chosen to be a power of two, as such a multiplication or division can be mapped to a shift operation in an embedded implementation. The last step in the signal energy computation was a moving average filter. 


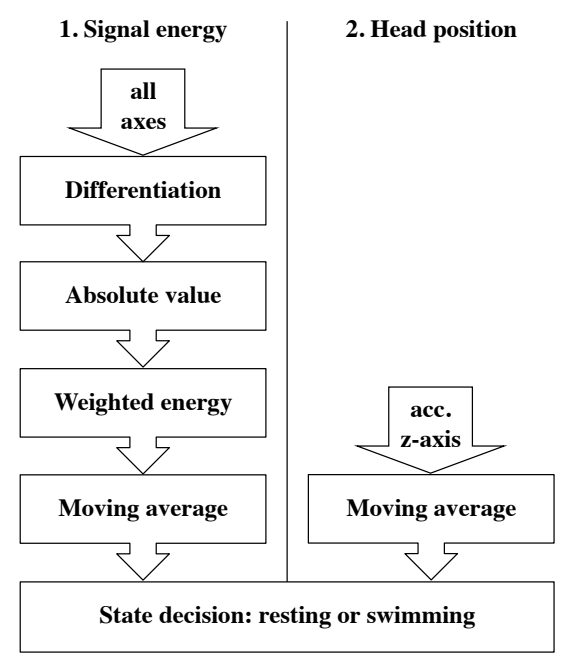

Fig. 3. Overview of the state detection based on signal energy and head position.

We used Knuth's computation [15] that is well suited for an embedded implementation and transforms the unfiltered signal $x(t)$ to the filtered signal $y(t)$. First, the difference of the last filtered value $y(t-1)$ and $x(t)$ was computed and scaled by the filter width (Eq. 3).

$$
\delta=(x(t)-y(t-1)) / \text { width } ;
$$

Second, this difference was used to determine the current filtered value $y(t)$ value based on $y(t-1)$ (Eq. 4).

$$
y(n)=y(t-1)+\delta
$$

We chose the filter width to be $2^{8}$ to be able to map the division to a shift operation. A threshold according to the labeled study data was defined that differentiated the resting from the swimming phase for most cases.

The head position defined the posture of the head and was employed to differentiate the resting phase from backstroke swimming. There, only minimal head movements were recorded and resulted in low values for the signal energy. The moving average algorithm described in Eq. 3 and Eq. 4 was applied to the z-axis of the accelerometer and a threshold separating the posture in backstroke swimming and resting was defined.

The result of the signal energy and the head position computation were combined to the final state decision. As postprocessing step in the state detection, we removed state changes that lasted shorter than one second.

2) Turn detection: Turns mark the end of a lane and are therefore important events to measure time and distance. We considered two criteria to detect turns, the head position and a classification system.

We tracked the same axis as for the state detection, the accelerometer z-axis, to detect turns bounding the lanes where the backstroke style was swum. A characteristic drop due to the turnaround of the head was registered to mark a turn.

In addition, we trained a classification system to determine the remaining turns that were not found with the head position criterium. Therefore, we manually segmented two seconds of each turning event for the TURN class. Furthermore, the remaining data, which were determined to be of the swimming state, were also split in 2 second intervals to make up the
SWIM class. The class distribution was skewed as the number of TURN events was much lower compared to the number of patterns in the SWIM class. We therefore doubled the number of TURN patterns by adding each pattern twice.

The two criteria were combined to a final turn detection that was checked every second. If a turn was registered, the detection was set inactive for eight seconds to avoid double detection. We employed a static duration of 1.5 seconds for each turn.

3) Swimming style classification: We set up a classification problem to determine the swimming style. Therefore, the manual turn segmentation was used to split a complete session in lanes. According to the protocol, the first lane was labeled with butterfly (BU), the second lane with backstroke (BA), the third lane with breaststroke (BR) and the last lane with freestyle (FR). We evaluated different interval sizes between 1 and 20 seconds for classification performance and processing effort.

4) Evaluation: The collected data was split in two distinct sets, a training set and a test set. The training set consisted of ten subjects with two swimming sessions each. One session was recorded before and one after the training session. The test set consisted of one subject with two swimming sessions (before and after) and one subject with one session (before). The classification results on the training set were determined with leave-one-subject-out cross-validation [16]. This method was chosen as multiple patterns of each subject contributed to the data sample. In addition, we expected subject-dependent characteristics in the data.

The classification results for the test set were determined with a training-test procedure. Therefore, a classifier was trained on the training set and tested on the previously unseen test set.

\section{RESULTS}

\section{A. Training data}

1) Turn classification: The training set for the turn classification consisted of 1721 patterns. As each swimming session consisted of three turns and was added twice to the training set, the TURN class consisted of 120 patterns. The SWIM class comprised of 1601 patterns accordingly.

The classification rate of the SWIM class was $99.6 \%$, the classification rate for the TURN class was $80.0 \%$. This resulted in an overall classification rate of $98.2 \%$. A distribution of misclassified patterns is shown in Tab. III.

To save the trained classification system on the target hardware, 98 floating point variables for the linear models and one integer value to store the pattern length were determined. The ECST also computed the number of operations that were needed for feature extraction and classification (Tab. IV).

TABLE III. CONFUSION MATRIX OF THE TURN CLASSIFICATION

\begin{tabular}{|c|c|c|}
\hline \multicolumn{2}{|c|}{ Prediction } & \\
\hline SWIM & TURN & Label \\
\hline 1594 & 7 & SWIM \\
\hline 24 & 96 & TURN \\
\hline
\end{tabular}

2) Swimming style classification: The size of the training set for the swimming style classification varied between 3160 patterns (1 sec interval) and 196 patterns $(20 \mathrm{sec})$. The interval 
TABLE IV. COMPUTATIONAL EFFORT OF THE TURN CLASSIFICATION IN NUMBER OF OPERATIONS

\begin{tabular}{ccc} 
Operation & Feature extraction & Classification \\
\hline,+- & 43,170 & 97 \\
$\times$ & 21,654 & 96 \\
$\div$ & 4,872 & 2 \\
$\sqrt{x}$ & 18 & - \\
$\leq$ & 4,788 & 5 \\
\hline \hline Overall & 74,502 & 200
\end{tabular}

of $13 \mathrm{sec}$ delivered the best result $(90.6 \%)$ and the $18 \mathrm{sec}$ interval the lowest classification result (84.3\%) (Fig. 4).

For all intervals, the memory demand of the classification system remained the same and was calculated with 196 floating point parameters and 1 integer value. To make the computational effort comparable across all intervals, we summed all operations and normalized the values to the number of operations per second. The results show an exponential decrease of the overall number of operations with increasing intervals sizes (Fig. 5). The most operations per second were needed in the 1 second interval $(37,704)$ and the fewest in the 20 second interval $(37,225)$.

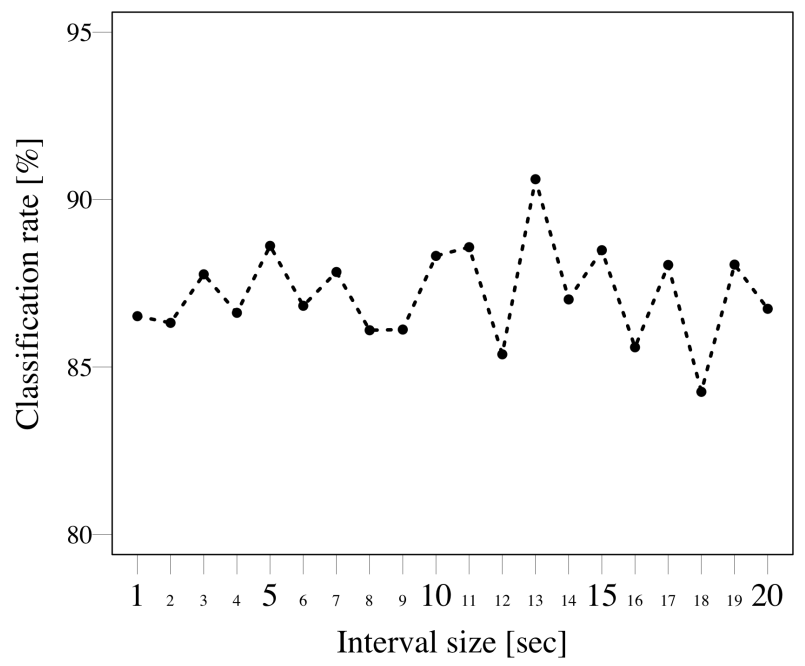

Fig. 4. Swimming style classification results for different interval sizes.

\section{B. Test data}

1) Turn detection: The turn classification detected eight out of nine turns of the test set. All 513 intervals where the subjects were swimming were classified as SWIM. Thus, the turn classification reached an overall classification rate of $99.8 \%$, and class-dependent classification rates of $100 \%$ for the SWIM class and $88.9 \%$ for the TURN class. The turn detection algorithm detected the single misclassified turn and therefore all turns of the test set were successfully determined without additional misdetections.

2) Swimming style classification: We used the intervals with the best classification rates on the training set for classifying the test set. These were the 5 second interval and the 13 second interval. For the segmentation of the swimming session, the automatic state and turn detection was used.

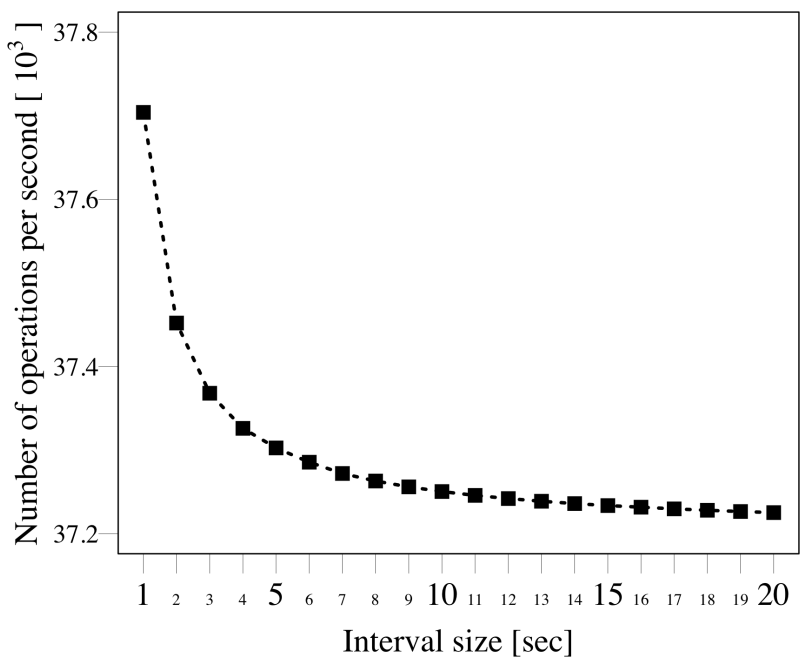

Fig. 5. Number of operations per second for classification systems with different interval sizes

The 5 second interval experiment classified the 100 test patterns of the three datasets with a classification rate of 95.0\%. Each lane was classified correctly when considering the majority vote over one lane. The five misclassifications were registered for the butterfly and breaststroke swimming styles.

In the 13 second interval, the 38 test patterns were classified with a classification rate of $92.1 \%$. Again, each lane was classified correctly with the majority decision. For each subject, one misclassification was registered either for the butterfly or the breaststroke style.

\section{DISCUSSION}

The analysis results demonstrate that determining turn events and swimming styles with a head-worn kinematic sensor is possible with high accuracy. This was shown for a cross-validated training set and confirmed with a disjoint test set for the four main swimming styles.

The test set analysis reached better results than estimated on the training set. This might be due to the size of the test set and due to the fact that the test set consisted solely of girls. However, the analysis on a disjoint test set confirmed the training results and proved the applicability to previously unseen subjects.

For the swimming style classification, we compared the results for different analysis interval sizes and the corresponding resource consumption. As the memory size of the trained classification system was constant, this aspect has no influence on a potential hardware decision. In contrast, the number of operations per second differed for different interval sizes and suggested choosing a long interval to minimize computational effort. Another aspect that might influence the interval decision was the decision granularity. Shorter intervals have a higher time resolution and are therefore suitable if the swimming style is changed during a lane or to give live feedback. In addition, more decisions during one lane alleviate single misclassifications and might result in a more robust classification of the swimming style of a lane. From a performance perspective, the classification rates of 
the considered intervals were between $84.3 \%$ and $90.6 \%$ and, thus, did not vary considerably. A small variation of less than 500 operations per second was also registered for the overall number of required operations. To sum up the performance and effort analysis for the swimming style classification, a trade-off between decision granularity and robustness on the one hand and resource consumption and performance on the other hand has to be made for a final system decision.

We presented a turn detection approach that combines head position tracking with a turn classification system. The advantage of the head position tracking is its computational ease as it consists of threshold comparisons. In contrast, the turn classification is computationally demanding and the major part of operations was used for feature extraction. Thus, feature reduction is the most promising approach to reduce the computational effort. Especially the features that require the most operations are a target for removal. We also propose to incorporate prior knowledge to the turn detection. This could be a sleep time after a detected turn that lasts for the minimum time needed to swim a lane.

We analyzed the swimming style misclassifications on the test set in more detail. We discovered that most of the misclassification occurred at the beginning or the end of a lane. Furthermore, the butterfly and the breaststroke class were confused most often. We hypothesize that misclassifications at the start or end of a lane occurred due to a beginning turn or diving phase. Turn preparation and diving phase detection has to be addressed in further research. We further assume that the confusion of butterfly and breaststroke appeared due to similar head movements of the swimming styles. This was already visible in the recorded data and seems to be confirmed by the classification result. Anyway, it can be stated that the butterfly and breaststroke showed the most similar head movement patterns across all swimming styles.

We provided a resource consumption analysis for parts of the data processing described. However, other aspects like the conversion of the analog-digital converter output in acceleration and rotational velocity units was missing. This has to be addressed in further research to compile a complete overview of computational effort and memory demand.

The results for the state and turn detection as well as the swimming style classification proved the applicability of the proposed data processing. We designed the algorithms under consideration of the computational complexity to enable the porting to an embedded microcontroller. However, an evaluation of the precise timing regarding the event detection is missing so far. Therefore, the recorded video has to be precisely aligned with the recorded inertial data to quantify delays in the event detection.

\section{SUMMARY AND OUTLOOK}

This article presented an analysis of kinematic swimming data recorded at the occiput. We conducted a research study to collect swimming sessions that consisted of the four main swimming styles and were collected in rested and in fatigued state. An energy-based state detection was used to detect whether the athlete was resting or swimming. Based on this decision a two step turn detection segmented single lanes. Finally, we trained a classification system that was able to distinguish the swimming styles with high accuracy.
The algorithms were designed under consideration of their computational complexity. Furthermore, practical cost measures were computed for the classification steps. This work is the basis for porting the presented swimming analysis system to an embedded microcontroller. This would be the next step towards an athlete support system that gives instant feedback about timing and distance of a swimming training. Due to the sensor positioning at the back of the head, the system is unobtrusive and offers the opportunity to give visual and acoustic feedback. Our vision is to integrate the feedback capabilities directly in the swimming goggles.

\section{ACKNOWLEDGMENT}

The authors would like to thank all volunteers who participated in the study and the SSG 81 Erlangen for supporting this research. This work was funded by the Bavarian Ministry for Economic Affairs, Infrastructure, Transport and Technology and the European Fund for Regional Development.

\section{REFERENCES}

[1] D. A. James, B. Burkett, and D. V. Thiel, "An unobtrusive swimming monitoring system for recreational and elite performance monitoring," in Proc. of the APCST 2011, Hong Kong, 2011, pp. 113-119.

[2] M. Bächlin and G. Tröster, "Swimming performance and technique evaluation with wearable acceleration sensors," Pervasive and Mobile Computing, vol. 8, no. 1, pp. 68-81, 2011.

[3] Tanya Le Sage, A. Bindel, P. Conway, L. Justham, S. Slawson, and A. West, "Development of a real time system for monitoring of swimming performance," in Proc. of the Conference of the International Sports Engineering Association (ISEA), Vienna, 2010, pp. 2707-2712.

[4] Y. Ohgi, H. Ichikawa, and C. Miyaji, "Microcomputer-based acceleration sensor device for swimming stroke monitoring," JSME Int J., Ser. C, vol. 45, no. 4, pp. 960-966, 2002.

[5] A. Callaway, J. Cobb, and I. Jones, "A Comparison of Video and Accelerometer Based Approaches Applied to Performance Monitoring in Swimming," Int J Sports Sci Coach., vol. 4, no. 1, pp. 139-153, 2009

[6] J. Pansiot, B. Lo, and G. Yang, "Swimming Stroke Kinematic Analysis with BSN," in Proc. of the BSN 2010, Singapore, 2010, pp. 153-158.

[7] M. J. Mcgrath and T. J. Dishongh, "A Common Personal Health Research Platform SHIMMER ${ }^{\mathrm{TM}}$ and BioMOBIUS ${ }^{\mathrm{TM}}$," Intel Tech J., vol. 13, no. 3, pp. 122-147, 2009.

[8] J. Klucken, J. Barth, P. Kugler, J. Schlachetzki, T. Henze, F. Marxreiter, Z. Kohl, R. Steidl, J. Hornegger, B. Eskofier, and J. Winkler, "Unbiased and mobile gait analysis detects motor impairment in Parkinson's disease." PloS one, vol. 8, no. 2, p. e56956, 2013.

[9] B. Eskofier, P. Kugler, D. Melzer, and P. Kuehner, "Embedded Classification of the Perceived Fatigue State of Runners: Towards a Body Sensor Network for Assessing the Fatigue State during Running," in Proc. of the BSN 2012, 2012, pp. 113-117.

[10] H. Niemann, Pattern Analysis and Understanding. Springer, 1990.

[11] Shimmer User Manual, Shimmer Research, 2012, Revision 2R.e.

[12] U. Jensen, M. Ring, and B. Eskofier, "Generic Features for Biosignal Classification," in Proc. of the Sportinformatik 2012 Symposium, 2012, pp. 162-168.

[13] E. Frank, Y. Wang, S. Inglis, G. Holmes, and I. H. Witten, "Using Model Trees for Classification,” Mach Learn., vol. 32, pp. 63-76, 1998.

[14] M. Ring, U. Jensen, P. Kugler, and B. Eskofier, "Software-based Performance and Complexity Analysis for the Design of Embedded Classification Systems," in Proc. of the ICPR 2012, Tsukuba, 2012, pp. 2266-2269.

[15] D. Knuth, The Art of Computer Programming: Sorting and Searching. Addison-Wesley, 1973.

[16] S. Theodoridis and K. Koutroumbas, Pattern Recognition. Academic Press, 2009. 